\title{
La retroalimentación como estrategia para mejorar el proceso de enseñanza-aprendizaje.
}

\section{Feedback as a strategy to improve the teaching-learning process.}

La retroalimentación como estrategia para el aprendizaje

Evelyn Juliana San Andrés Soledispa ${ }^{(1)}$

Fanny Magaly Macías Figueroa ${ }^{(2)}$

Gabriela Lisseth Mieles Pico ${ }^{(3)}$

(1) Universidad Católica del Ecuador. e-mail: esan1717@ pucesm.edu.ec

ORCID: https://orcid.org/0000-0003-2464-4663

(2) Universidad Católica del Ecuador. e-mail: fmacias7045@pucesm.edu.ec

ORCID: https://orcid.org/0000-0002-8722-049X

(3) Universidad Católica del Ecuador. e-mail: gmieles9598@pucesm.edu.ec

ORCID: https://orcid.org/0000-0001-9942-1355

Contacto: esan1717@ pucesm.edu.ec

\section{Resumen}

El presente artículo tiene por objetivo analizar la retroalimentación como estrategia para mejorar el proceso de aprendizaje de los estudiantes de educación básica superior. Se trabajó un enfoque de investigación de tipo mixto. También se utilizó el método exploratorio y bibliográfico. Se empleó la técnica de la encuesta con resultados que indican que los docentes, la utilizan con la finalidad de reforzar las habilidades y destrezas de los estudiantes, emplean como estrategias la motivación y la autorreflexión. Se realizó un análisis de la evaluación del aprendizaje y estrategias. Se concluye que es un elemento efectivo, que aporta significativamente en el proceso de enseñanza - aprendizaje y mejora el aspecto formativo de docentes y estudiantes.

Palabras claves: Evaluación del aprendizaje, estrategias de aprendizaje, retroalimentación educativa, desempeño académico. 


\section{Summary}

The present article aims to analyze feedback as a strategy to improve the learning process of students in basic higher education. A mixed type research approach was used. The exploratory and bibliographic method was also used. The survey technique was used with results that indicate that teachers use it in order to reinforce the skills and abilities of students, use motivation and selfreflection as strategies. An analysis of the evaluation of learning and strategies was carried out. It is concluded that it is an effective element that contributes significantly to the teaching-learning process and improves the educational aspect of teachers and students.

Keywords: Learning assessment, learning strategies, educational feedback, academic performance.

\section{Introducción}

Ser docente es una carrera de mucha pasión y entrega, hacia lo que uno quiere, para esto se desarrollan estrategias educativas que permitan alcanzar los objetivos planteados con los estudiantes, el proceso de la evaluación en los educandos permite verificar la adquisición de conocimientos y consolidación de destrezas en cada uno de ellos, considerando los aspectos cualitativos y cuantitativos como punto imprescindible en el proceso de la retroalimentación, elemento clave para generar aprendizajes significativos.

La evaluación educativa se constituye un rol, que necesariamente debe estar presente en los procesos de enseñanza-aprendizaje, permitiendo elevar el nivel educativo de los educandos y la reflexión profunda sobre la práctica docente (Cruz \& Quiñones, 2012). Realizar la retroalimentación a los estudiantes fortalece las dificultades, de tal manera, que el docente ejecute nuevas herramientas eficaces para promover una mayor comprensión, no solo persigue que se lo establezca dentro del aula, en una asignatura en particular, sino en todo el proceso formativo.

En la Ley Orgánica de Educación Intercultural del 2012, en su artículo 11, sobre las obligaciones del docente establece "Dar apoyo y seguimiento pedagógico a las y los estudiantes, para superar el rezago y dificultades en los aprendizajes y en el desarrollo de competencias, capacidades, habilidades y destrezas;” (Asamblea Nacional, 2012, pág. 27), el desempeño del docente en su accionar logra que los educandos adquieran aprendizajes a partir de los procesos de reflexión sobre lo que pueden mejorar. 
El docente debe orientar, motivar e invitar al estudiante a la reflexión profunda de los aprendizajes que deben lograr, aquellos que ha adquirido y qué debe hacer para aprender de forma significativa, logrando los objetivos planteados (Cedeño \& Moya, 2019), aplicar la motivación al momento de impartir sus clases, permite lograr los objetivos planteados y que la intervención sea significativa para los estudiantes, se logra tener una visión clara en lo que se está fallando y como mejorar la práctica educativa.

El presente artículo tiene por objetivo analizar la retroalimentación como estrategia para mejorar el proceso de aprendizaje de los estudiantes de educación general básica superior, es importante conocer este estudio para descubrir pruebas netamente reales de la aplicación de estrategias pedagógicas de docentes y su entrega total a las actividades educativas.

\section{Material y métodos}

El enfoque de investigación que se aplicó es de tipo mixto, con características cuantitativas para analizar la realidad objetiva; así también, lo cualitativo, al buscar la profundidad de los significados, apuntando a la riqueza interpretativa.

Se empleó para la investigación el método exploratorio-bibliográfico; para interpretar conceptos, validar puntos de vista de varios autores y obtener criterios reveladores.

Se contó con una población de 20 maestros, tomando como muestra la totalidad de los mismos, además, el método mixto secuencial explicativo, que busca la recolección de datos en dos fases: se llevó a cabo lo cuantitativo, su posterior análisis y se usó los resultados obtenidos para planificar la fase cualitativa.

Para realizar la recolección de la información se empleó la técnica de la encuesta. Como instrumento se empleó el cuestionario y se aplicó a través de la web, que se usó con docentes para conocer el tipo de evaluación que aplican, el grado de motivación y el proceso de retroalimentación que llevan a cabo.

\section{La evaluación del aprendizaje}

La evaluación del aprendizaje, es una actividad que en la mayoría de los casos se realiza al final del proceso de aprendizaje de los educandos, para obtener información de las habilidades adquiridas; ésta debe ser un eje dinamizador e integral en el proceso enseñanza-aprendizaje, que no solo debe estar presente al término de una etapa de instrucción, más bien, brinda la oportunidad de reforzar las 
destrezas que no se han alcanzado y al docente le proporciona la ocasión para volver a enseñar lo que no ha quedado claro (Arredondo, Diago, \& Cañizal, 2010).

En este sentido se debe aplicar la evaluación educativa durante todo el proceso de enseñanza de manera integral para lograr obtener resultados que permitan establecer las fallas del proceso, para luego realizar una retroalimentación en los aprendizajes que no se hayan logrado adquirir. Los aprendizajes que obtengan los educandos deben ser significativos; para fomentar la creatividad y formar personas activas que sean parte de la solución de los problemas de la sociedad actual (Moreira, 2012); para lograr esto, se necesita evaluar durante el proceso de la formación, tanto conocimientos previos como nuevos.

Para obtener mejores resultados es necesario realizar un análisis en el evaluador como en el evaluado de manera simultánea; este proceso constituye una vía de comunicación interpersonal, que cumple todas las características y presenta todas las complejidades; donde los papeles de los involucrados pueden alternarse e incluso simultáneamente, de esta manera se obtienen resultados tanto del objeto como del sujeto evaluado para determinar características y peculiaridades entre sí (Freire, 2017).

La evaluación de aprendizaje como actividad genérica, proporciona resultados para obtener un juicio sobre la apreciación auténtica que se realiza, se establece que tiene por objetivo valorar los conocimientos, los procesos y los resultados, con propósitos ya establecidos (González, 2001). Refiriéndose al papel que desempeña en la sociedad, en la institución, en el proceso de enseñanzaaprendizaje para los individuos implicados en éste; sin embargo, en algunas ocasiones no se valora, ni se cumplen los propósitos para los cuales se realiza, dejando una brecha entre lo adquirido y la función que tiene en el ámbito educativo.

Actualmente se reconoce a la evaluación como uno de los puntos privilegiados para estudiar el proceso de enseñanza - aprendizaje, ya que, se demuestra al profesorado los diferentes enfoques de los cuales se debería trabajar de una mejor manera, en la figura 1 se muestran las funciones de la evaluación.

La evaluación se reconoce actualmente como uno de los puntos privilegiados para estudiar el proceso de enseñanza-aprendizaje, abordarlo como dificultad, supone necesariamente tocar todos los problemas fundamentales de la pedagogía (Estebaranz, 2016), cada aspecto que exista, debe adquirir más conciencia para poder analizar de mejor manera el saber impartido. 
En lo relativo a las estrategias de aprendizaje, se han notificado varios estilos de aprendizaje, que generan una amplia gama de estrategias, que incrementa el nivel educativo en todos los aspectos, además, aporta con la sociedad, en los últimos años ha mejorado, contando con las herramientas necesarias para cambiar el estilo de enseñanza tradicional a una crítica, reflexiva, esto también ayuda de manera significativa al proceso de formación del estudiante.

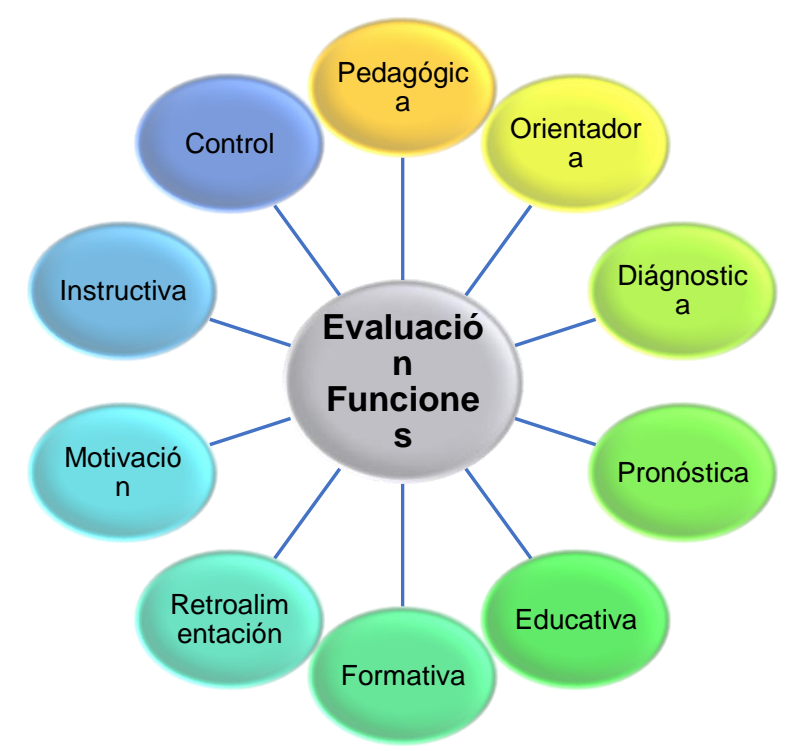

Figura 1. Funciones de la Evaluación interrelacionadas con la enseñanza y el aprendizaje. Fuente: (Freire, 2017)

Por otra parte al hablar del aprendizaje contempla varios diseños, modelos, tipos, basados en la adquisición de nuevos conocimientos que ayuden al estudiante a mejorar su capacidad intelectual, el aprender algo o conocer algo distinto, permite asimilar los diferentes problemas que acarrean la sociedad y poder interiorizarlos causando una reflexión para el proceso, mediante éstos se suman o modifican habilidades, valores, conductas, destrezas, como efecto de la experiencia, estudio, instrucción o razonamiento (Zapata, 2012).

\section{Estrategia de aprendizaje}

Las estrategias son mecanismos para lograr la adquisición de destrezas de los estudiantes, utiliza una secuencia pedagógica que abarca: inicio, desarrollo y final; tomando en cuenta los objetivos que de desean alcanzar; la correcta aplicación de estos mecanismos facilitan la recuperación de conocimientos y la secuencia dinámica de los mismos; son necesarios porque le proporcionan a los educandos una organización gráfica de los contenidos para su rescate inmediato, cuando lo necesiten (Prieto, 2012). 
Una estrategia de aprendizaje es un mecanismo que ayuda a mejorar las capacidades de un estudiante, éstas fortalecen las macro - actividades relacionadas con el acto de aprender, como: atención, comprensión, transferencia, reproducción; se descubren conocimientos mediante la observación, imitación y construcción de un conocimiento crítico e interpretativo en base al modelado proporcionado (Llera, 2003). En la figura 2, se muestra la función de las estrategias de selección, organización y elaboración en la construcción de los conocimientos.

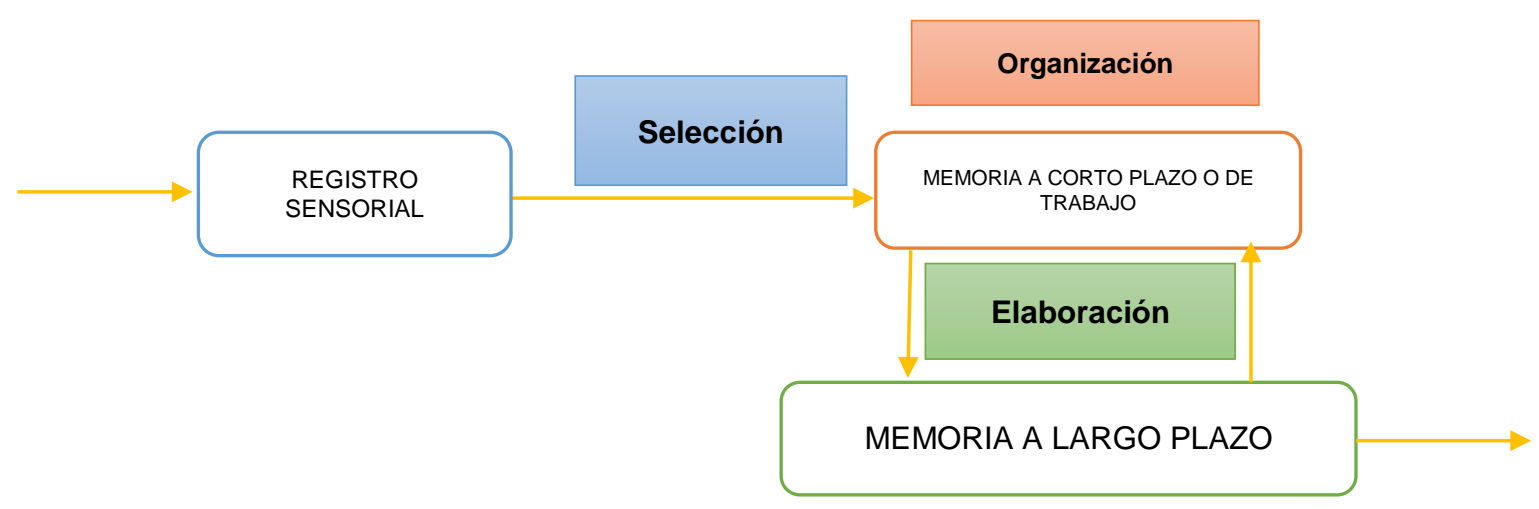

Figura 2. Función de las estrategias en la construcción de los conocimientos. Fuente (Llera, 2003)

Existe la necesidad de conocer que no todos los estudiantes poseen las mismas capacidades y habilidades para aprender, no obstante, el mecanismo que se emplea debe ser eficaz y muy acertado para cada uno, de hecho, no solo la estrategia debe ser motivo de una aplicación estricta, también, se puede adaptar a las necesidades de los educandos.

Cabe destacar que detrás del desempeño académico está la asertividad de estrategias, metodologías, recursos didácticos que aplica el docente en sus clases; más las condiciones de la institución e incluso la eficacia del sistema educativo. Se valora en términos tanto cualitativos como cuantitativos los conocimientos que el estudiante ha adquirido en el proceso de formación integral y como éste desempeño se ve reflejado en el accionar del educando (Quinteo \& Vallejo, 2013), en muchos contextos solo es valorado por una calificación, más no, por las destrezas que ha alcanzado, que se ve reflejada en sus criterios y acciones.

La utilización de estrategias efectivas, están relacionadas con alcanzar mejoras en el desempeño académico, la evaluación de aprendizajes, mostrando el dominio de destrezas y la reflexión que pueda tener el estudiante de acuerdo a la temática interiorizada. 
Esta estrategia tiene como propósito, dirigir, acompañar y estimular el aprendizaje permanente en los estudiantes, sirve de apoyo, apuntando a una evaluación con carácter constructivo y prospectivo, para desarrollar la autorreflexión y autoevaluación del proceso. Lograr un resultado integral significa analizar el actuar del profesorado, en cuanto a lo pedagógico y lo práctico, considerando que ésta ayuda alcanzar el carácter formativo (Canabal \& Margalef, 2017).

En este sentido la retroalimentación, es considerada como un aporte al desempeño de los estudiantes, convirtiéndose en información entregada por el agente, que es el docente; es una consecuencia del trabajo del educando; estos datos o comentarios deben ser claros, significativos y comprensibles, logrando que los escolares relacionen los conocimientos previos, establezca conexiones lógicas y generen aprendizajes significativos (Hattie \& Timpeley, 2007).

La retroalimentación que reciben y proveen los estudiantes, debe estar directamente relacionada con ayudarlos a aprender, debe utilizarse de manera pedagógica los implementos necesarios para que así, tengan un mejor desempeño académico (Wiliam, 2012). Se considera como un proceso que suministra información, tanto a educandos como docentes, permitiendo que se modifique el transcurso de enseñanza - aprendizaje de manera inmediata convirtiéndose como herramienta eficaz, siempre y cuando, se utilice de manera objetiva y con indagación directamente relacionada a la misma.

Para que esta sea efectiva, todas las personas involucradas deben estar convencidas de que los cambios en la ruta del aprendizaje se pueden lograr y se sientan motivadas a esforzarse. Para fomentar una retroalimentación efectiva, el salón de clases tiene que ser un ambiente donde los errores no sean vistos como algo crítico, sino un ambiente donde estos sean considerados oportunidades para seguir creciendo y mejorando, se debe tener cuidado con el manejo de los aspectos positivos y negativos en el estudiante (Ellis \& Loughland, 2017).

Entre los tipos de retroalimentación se pueden mencionar: la retroalimentación positiva, que resalta lo que se ha realizado acertadamente; negativa, da a conocer los aspectos que no se han logrado y bipolar cuando además de lo bueno y malo se motiva a mejorar; la última es la más recomendada (Cedeño \& Moya, 2019).

La retroalimentación entre compañeros está presente de manera constante en la clase y fomenta a que los estudiantes sean recursos de aprendizaje entre ellos mismos. El rol del docente en este caso es ser de ejemplo y mostrar la manera en que los estudiantes deberían interactuar entre sí. 
Gracias a la evidencia que proporciona la retroalimentación, se pueden reconocer: el impacto, las fortalezas y aspectos a mejorar del proceso enseñanza aprendizaje; además, es una forma en la que los docentes pueden visualizar el proceso de aprendizaje, métodos de trabajo y cómo perfeccionarlo, también busca la formación de un estudiante reflexivo, autónomo, que persigue constantemente su desarrollo y autoformación (Valdivia, 2014).

\section{Resultados}

Los resultados reflejan en el análisis del proceso de retroalimentación como estrategia para mejorar el proceso enseñanza de los estudiantes de básica superior.En la encuesta diseñada se obtuvieron resultados significativos y se analizó el proceso de retroalimentación como una estrategia para mejorar el proceso enseñanza de los estudiantes de básica superior. En la encuesta aplicada se corroboró que el 100\% de los docentes manifiestan que usan la retroalimentación, en el proceso de evaluación.

En cuanto a los tipos de retroalimentación, en la figura 3 se observa que la más utilizada por los docentes, es la bipolar en un $60 \%$, seguida de la positiva en un $40 \%$.

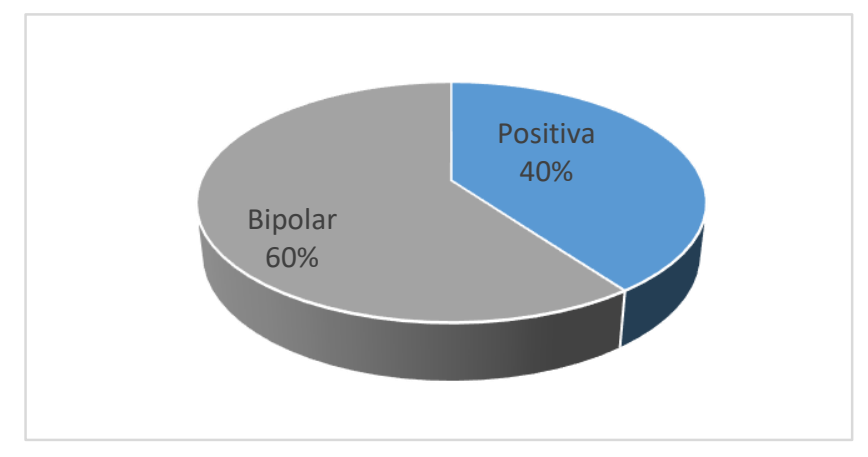

Figura 3. Tipo de Retroalimentación

Entre las estrategias que utilizan los docentes en el proceso de retroalimentación destacan que el $33 \%$ utiliza la motivación, un 26\% utiliza generar la autorreflexión en los estudiantes, el 22\% escogió el análisis aspectos positivos y negativos en el proceso, un 13\% envían tareas extras, un 6\% realizan reuniones personalizadas, esto se observa claramente en la figura 4.

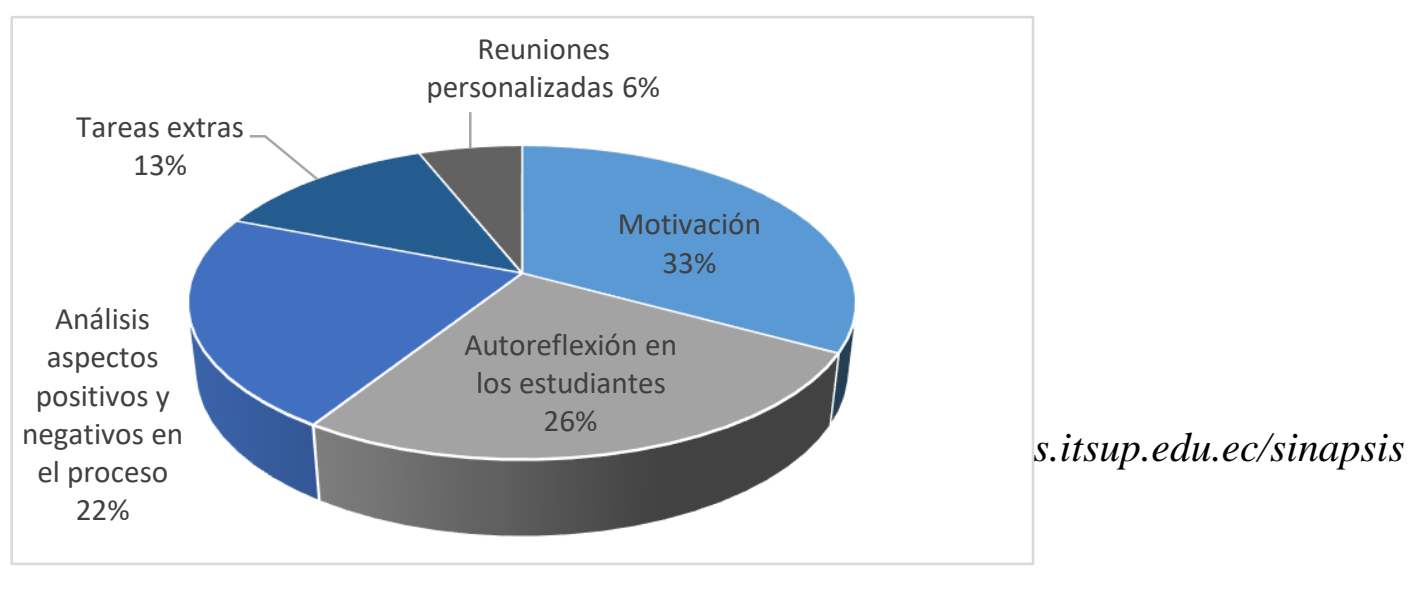


Figura 4. Estrategias para la retroalimentación

El objetivo que se persigue con la retroalimentación, el 90\% manifestó que buscan reforzar las habilidades, destrezas y conocimientos, $10 \%$ intenta mejorar una calificación, esto se evidencia en la figura 5 .

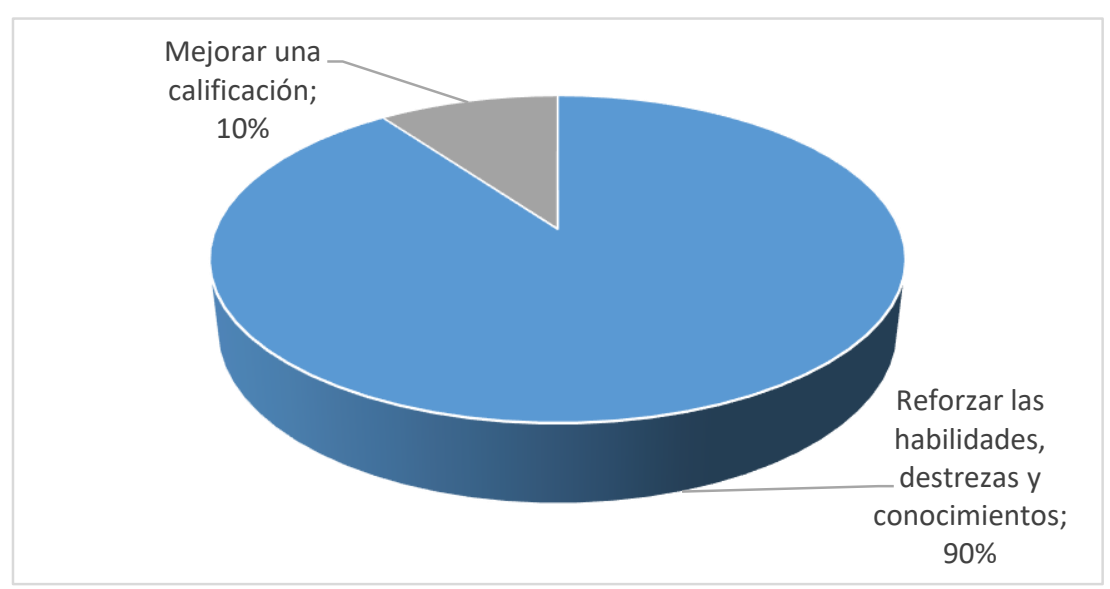

Figura 5. Objetivo de la Retroalimentación

\section{Discusión}

Dentro de la enseñanza-aprendizaje se activa el proceso de la evaluación como método referencial para la verificación de adquisición de conocimientos y la consolidación de destrezas de cada uno de los individuos. La evaluación de aprendizaje proporciona resultados para obtener un juicio, sobre la apreciación auténtica que se realiza, tiene como meta valorar conocimientos, procesos y resultados, con propósitos ya establecidos, permitiendo determinar características y particularidades del proceso evaluativo, en el estudio se concuerda (González, 2001).

Dentro del proceso educativo surge la retroalimentación, que actúa de la mano con la evaluación, éste, tiene como propósito dirigir, acompañar, estimular el aprendizaje permanente en los estudiantes, sirve de apoyo y apunta a una evaluación con carácter constructivo - prospectivo, para desarrollar la autorreflexión y autoevaluación (Canabal \& Margalef, 2017).

El presente proyecto de investigación tiene como objetivo analizar la retroalimentación como estrategia para mejorar el proceso de aprendizaje de los estudiantes de EGB Superior, debido a que el instrumento utilizado para la recolección de información fue la encuesta que se aplicó a 20 docentes, a continuación, se muestra el análisis de los resultados obtenidos con un contraste desde las aseveraciones de autores. 
Se pudo evidenciar que el $100 \%$ de los docentes manifiestan que durante el proceso de enseñanza aprendizaje, aplican la retroalimentación, una vez realizado la evaluación, para fortalecer los conocimientos adquiridos. Se confirma que está práctica directamente está relacionada con ayudar a los estudiantes a aprender, utilizando de manera pedagógica los implementos necesarios para que así, los educandos tengan un mejor desempeño académico (Wiliam, 2012).

Entre los tipos de retroalimentación un $60 \%$ de los docentes utilizan la bipolar, seguida de la positiva con un $40 \%$. Los autores afirman que la segunda, es cuando se resalta lo acertado del proceso y la primera es cuando además de los dos aspectos, hay una estimulación para mejorar. Lo anterior se fortalece con el hecho de que los maestros aplican en un 75\% la motivación y en un $60 \%$ indican que dentro del proceso generan la autorreflexión en los estudiantes, el intercambio de saberes y con esto se logra alcanzar aprendizajes significativos (Cedeño \& Moya, 2019).

A partir de esta experiencia, este estudio aporta en gran proporción al fortalecimiento del proceso enseñanza-aprendizaje, porque, crea en el maestro una visión que apunta a un tipo de evaluación formativa, permite orientar de manera oportuna a los estudiantes y dar énfasis de manera significativa a su proceso cognitivo; es importante que se continúe con este proceso de investigación, para fomentar la retroalimentación en todas las instituciones y contrastar la información proporcionada por los docentes con las opiniones de los educando y que no simplemente sirva para mejorar una calificación.

\section{Conclusiones}

La evaluación en el educando evidencia los conocimientos adquiridos y los resultados obtenidos de una evaluación integral, permiten aplicar la retroalimentación para mejorar el aspecto formativo de los involucrados, a través de estrategias de motivación y autorreflexión como parte del proceso para la construcción de los aprendizajes significativos, ésta desarrolla habilidades, destrezas en los educandos.

La retroalimentación es un elemento efectivo dentro del proceso de enseñanza -aprendizaje como reemplazo a las críticas destructivas, que desmotivan a los aprendices, debe de ser tomada como un aspecto formativo para lograr una educación integral en los estudiantes.

\section{Bibliografía}


Arredondo, S., Diago, J., \& Cañizal, A. (2010). Evaluación educativa de aprendizajes y competencias. Madrid: Pearson Educación. Recuperado de: https://bit.ly/2Pg9aZq

Asamblea Nacional. (2012). Ley Organica de Educacion Intercultural. Quito.

Canabal, C., \& Margalef, L. (2017). La retroalimentación: la clave para una evaluación orientada al. Profesorado. Revista de Currículum y Formación de Profesorado, 21(2), 149170. Recuperado de https://www.redalyc.org/pdf/567/56752038009.pdf

Cedeño, E., \& Moya, M. (2019). La retroalimentación como estrategia de mejoramiento del. Atlante. Recuperado de https://www.eumed.net/rev/atlante/2019/08/retroalimentacioneducandos.html

Constituyente, Asamblea. (2008). Constitución del Ecuador. Montecristi.

Cruz, F., \& Quiñones, A. (2012). Importancia de la evaluación y autoevaluación en el rendimiento académico. Zona proxima, 96-104.

Ellis, J., \& Loughland, T. (2017). 'Where to next?' Examining feedback received by teacher education students. Issues in Educational Research, 27(1), 51-63. Recuperado de http://www.iier.org.au/iier27/ellis.html

Estebaranz, J. (2016). LA EVALUACIÓN DE LOS APRENDIZAJES. Profesorado- Revisata de curriculum y formacion del profesorado, 21(4), 381-404.

Freire, E. (2017). La evaluación del aprendizaje en la Educación Superior. Universidad y Sociedad, 9(5), 90-96.

González, M. (2001). La evaluación del aprendizaje. . Tendencias y reflexión crítica. Educ Med Super, 15(1), 47-62. Obtenido de:

http://scielo.sld.cu/scielo.php?script=sci_arttext\&pid=S0864-21412001000100010

Hattie, J., \& Timpeley, H. (2007). The Power of Feedback. Review of Educational Research, 77(1), 81-112. Recuperado de https://journals.sagepub.com/doi/abs/10.3102/003465430298487

Ley Organica de Educacion Intercultural. (2012). Ecuador.

Llera, J. (2003). Estrategias de aprendizaje. Revista de educación, 55-73. Recuperado de http://www.educacionyfp.gob.es/dam/jcr:0bc115bf-2ee5-4894-91f5-7e32e07059d4/re3320411443pdf.pdf

Moreira, M. (2012). ¿ Al final, qué es aprendizaje significativo? Curriculum, 29-56. Recuperado de: http://riull.ull.es/xmlui/handle/915/10652

Prieto, J. (2012). Estrategias de enseñanza-aprendizaje. México: Pearson educación. Recuperado de: htps://cmapspublic2.ihmc.us/rid=1V2J9XZYF-B98X90-3vqc/ estrategias\%20de\%20ense\%c3\%91anza-aprendizaje.pdf 
Quinteo, M., \& Vallejo, G. (2013). El desempeño académico: una opción para la cualificación de las instituciones educativas. Plumilla educativa, 93-115. Recuperado de https://dialnet.unirioja.es/servlet/articulo?codigo $=4756664$

Valdivia, S. (2014). Retroalimentación efectiva en la enseñanza universitaria. En Blanco y Negro, 5(2), 20-24. Recuperado de http://revistas.pucp.edu.pe/index.php/enblancoynegro/article/view/11388

Wiliam, D. (2012). Feedback: Part of a System. Feedback for Learning, 30-34. Recuperado de http://hoelscherb.pbworks.com/w/file/fetch/58702168/Feedback- Part_of_a_system.pdf

Zapata, M. (2012). Teorías y modelos sobre el aprendizaje en entornos conectados y ubicuos. Departamento de Computación, Universidad de Alcalá, España, 2-49. Obtenido de http://eprints.rclis.org/17463/1/bases_teoricas.pdf 